\title{
Structural pathology of the endocrine organs of calves with cryptosporidiosis
}

\author{
Ildar Zalyalov, Orazali Mullakaev, Evgeny Kirillov*, and Elena Zaikina \\ Kazan State Academy of Veterinary Medicine, Kazan 420029, Russia
}

\begin{abstract}
It is generally accepted in practical veterinary medicine that cryptosporidiosis invasion of the gastrointestinal tract of calves is a local pathological process and the disease outcome is completely determined by the local etiological factor. Pathomorphological changes in the gastrointestinal tract, detected during the invasion, and metabolic disorders in the body of sick calves cannot occur without changes in the structure of adaptive mechanisms. Therefore, studies of the endocrine organs reaction in acute and chronic forms of the cryptosporidiosis course have been carried out. It has been found that calves during the neonatal period have the acute course of invasion accompanied by severe violations of the most functionally loaded structures of the endocrine organs. This is manifested in the form of increased mechanisms of programmed necrosis, the occurrence of reversible and irreversible degenerative processes, and local hemocirculation disorders. The pathological metamorphosis arising from cryptosporidiosis infection in endocrinocytes led to significant violations of the adaptive systems of the calves' body and ultimately ended in an unfavorable disease outcome. Pathological changes with a predominance of atrophic, sclerotic, and necrobiotic processes in the chronic course of invasion were manifested in all structures of the studied endocrine organs simultaneously. A prolonged state of structural and functional overstrains of the endocrine organs eventually led to the depletion of adaptive hormone resources. The pluriglandular insufficiency state in the body was crucial in the pathogenesis and outcome of the chronic course of cryptosporidiosis in calves.
\end{abstract}

\section{Introduction}

Most studies in the pathomorphology of cryptosporidiosis are devoted to the study of the gastrointestinal tract structure of sick animals, while the reactions of the endocrine system in the disease remain insufficiently explored [1-10]. In this connection, pathohistological studies of the endocrine organs of calves with a chronic form of cryptosporidiosis have been carried out.

\section{Material and research methods}

Studies on the endocrine system microstructure of calves' organs during the neonatal period with acute and chronic forms of cryptosporidiosis were performed on the materials taken from livestock farms in Buinskii, Arskii, Verkhneuslonskii, Vysokogorskii, KamskoUstinskii regions of Tatarstan in 2018-2019. Symptoms of acute and chronic catarrhal gastroenterocolitis were established by the pathomorphological autopsy results of calves. Cryptosporidium oocysts were found in impression smear of feces of sick calves by sedimentation in a formalin-ether mixture and staining with carbol fuchsin according to Ziehl-Neelsen. The presence of Cryptosporidium parvum was determined by the immunoenzyme method in feces of sick calves.

Particles of the adrenal, thyroid and pancreas glands were selected for histological examination at autopsy of sick calves. Pathological material was fixed in a $10 \%$ solution of neutral formalin, dehydrated with ethanol, and compacted in chloroform-paraffin and paraffin. Sliced sections of $8 \mu \mathrm{m}$ thick were stained with hematoxylin and eosin. Histological sections of organs were examined under a light microscope. The morphometric parameters of the histological structures in the examined organs were determined using the eyepiece nozzle MOV (ocular spiral micrometer) 1.5. Statistical analysis of the obtained digital material was performed using Microsoft Excel.

\section{Results}

Exocrine pancreas cells in calves with an acute course of cryptosporidiosis were distinguished by a sharp decrease in the volume of the cytoplasm apical region. They contained only single zymogen granules near the cytolemma due to the weakening of the biosynthesis of digestive enzymes. Most of the decreasing acinar cells lost their pyramidal structure. The basal and apical regions of these cells became unclear marked. The

\footnotetext{
* Corresponding author: kirilloff.eug@ yandex.ru
} 
lumen profiles of the intra- and interlobular excretory ducts of the gland were significantly narrowed. The islet apparatus of the gland was also distinguished by a decrease in volume and a variety of forms. Most exocrinocytes in the islets had a rounded, oval cytoplasm form. The nuclei of these cells as a result of hypofunction were also reduced in volume, and their karyoplasm, saturated with heterochromatin, had a sharply basophilic form. Poorly marked small nucleoli located in close proximity to the karyolemma also characterized a reduced level of RNA biosynthesis in these cells.

The islet apparatus of the gland in calves with an acute form of cryptosporidiosis was distinguished by polymorphism, a decrease in volume, and sparseness of its cellular composition. $\beta$ cells prevailed among the endocrinocyte population. They were small, round, oval, sparsely polymorphic, with sharply oxyphilic, giving a devastated form to the cytoplasm. The nuclei in these cells were distinguished by hyperchromia and a reduced volume. The described insulocytes in their pathomorphological characteristics corresponded to cells in a state of hypofunction. Single $\alpha$-cells were located among $\beta$-cells, mainly along the periphery of the islets. They were distinguished by a large volume of rounded cytoplasm and the presence of a nucleus containing functionally active euchromatin in the karyoplasm and a nucleolus located in the center. Round-oval Dendocrinocytes were found only in individual islets and always along their periphery. Hemocapillary lumen profiles were designated between endocrinocyte cords, as a result of previous intensive excretion of hormonal products by cells of the islet apparatus in the latter.

Signs of pathological metamorphosis noted in the pancreas, in exo- and endocrinocytes, manifested by signs of weakened biosynthesis and excretion of digestive enzymes, and hormonal products corresponded to the acute hypofunctional state of the gland. The weakening of the insulin production by $\beta$-cells was aimed at compensating for the phenomena of increasing energy deficiency in the organs and tissues of sick calves.

Calves suffering from chronic cryptosporidiosis had a prevalence of atrophic, dystrophic changes in the parenchyma of the exo- and endocrine apparatus of the organ in the pancreas. These calves had a prolonged course of the disease, which led to a more significant decrease in the volume of acinar cells. Most of them lost granular material in the zymogen zone. The desolation of the lumen profiles of the pancreas excretory ducts was objectively characterized by a sharp weakening of the digestive enzymes excretion. The endocrine gland apparatus in sick calves was distinguished by a sharp decrease in volume and sparseness of its cells. $\beta$-cells prevailed among a sparse population of sparse endocrinocytes, being small, round-oval in shape, with a sharply oxyphilic empty cytoplasm. The nuclei of these cells were characterized by pycnomorphism, the predominance of condensed heterochromatin. A small nucleolus was poorly visible in most of these nuclei. Consequently, insulin production also weakened in the islet apparatus of the gland during the chronic course of cryptosporidiosis. Single $\alpha$-cells were located among $\beta$ cells, mainly along the periphery of the islets. They were distinguished by a somewhat larger volume of rounded cytoplasm and also by the predominance of functionally inactive heterochromatin in the karyoplasm. Round-oval D-endocrinocytes were found only in individual islets and always along their periphery. Prolonged rarefaction of islet endocrinocytes populations of the glandular apparatus combined with a weakening of intra organ hemocirculation.

Sick calves with acute cryptosporidiosis in the adrenal gland had significant structural changes, primarily affecting all parenchyma and stromal cells. The medullary region reactively significantly expanded as a result of a pronounced acute infectious process in the gastrointestinal tract, mainly due to severe hyperemia of the venous sinuses. As a result, the ratio of the thickness of the cortical and cerebral substances leveled off and amounted to $1: 1$. The resulting structural restructuring of the adrenal glands of sick calves was determined by the mobilization of all the reserve capabilities of the cortex and medulla of the organ. First of all, it was manifested by an increase in signs of functional activation of parenchyma cells of the fascicular zone. Significant thickness of this zone cortex reaching $131.4 \pm 12.7 \mu \mathrm{m}$ indicated this. As a consequence of the previous active elimination of glucocorticoids, endocrinocytes with a foamy cytoplasmic structure were not detected in the inner fascicular zone. Cells with signs of development and completion of apoptosis were visible among adrenocorticocytes in this zone. Single spongiocytes were found among cells of the outer fascicular zone. Small cells in size and number lost the polygonal structure of the cytoplasm were located in some places in this zone with signs of apoptosis in the form of hyperchromia, deformation of cells and the presence of small bodies near them. In addition, cells with signs of necrobiosis in the form of hyperchromic nucleus particles took place.

Marked changes in the fascicular zone were reflections of the organ functional overstrain. The increase in signs of adrenocorticocytes apoptosis from the periphery deep into the fascicular zone corresponded to the natural flow of cortical cells renewal. The resulting decrease in catecholaminosynthetic cells corresponded to acute adrenocortical insufficiency.

The lowest level of severity of proliferative and biosynthetic activity was observed in cells forming a poorly defined glomerular zone of the cortex. The width of this zone was $9.7 \pm 1.2 \mu \mathrm{m}$. Small glomeruli in sick calves were formed from small cells of a rounded shape with rare inclusions of single petty lipid drops. Cells with signs of mitosis were not found in this cortical zone. The paucity and low mitotic and synthetic cells activity of the cortex glomerular zone indicated a sharp decrease in the level of synthesis and excretion of mineral corticoids in hemocirculation. The weakening mechanism of sodium ion retention in the kidneys of calves with an acute course of cryptosporidiosis, contributed to the increase in the severity of body tissues exicosis. The presence of small groups of cambial cells 
with oval or oblong nuclei and the thinnest rim of the cytoplasm, surrounded by rare reticulocytes and fibroblasts, was noted only in places in the regenerative subcapsular zone of the adrenal cortex. Subcapsular blood vessels were weak in repletion. The intermediate zone between the glomerular region and fascicular zone, due to the delay in the regenerative process, was poorly indicated by rare small cells with basophilic stained cytoplasm.

The reticular zone also had signs of underdevelopment, as evidenced by morphometric parameters; its thickness was $7.2 \pm 0.7 \mu \mathrm{m}$. It was formed by cells small in number with a basophilic staining of the cytoplasm containing no lipid inclusions and separated from a well-defined medulla. An inner capsule was uneven in thickness, consisting of thin bundles of collagen and reticulin fibers. Reticulocytes partly formed syntycyclic structures. The part of the reticular zone adrenocorticocytes penetrated into the fascicular zone creating an uneven transition of cells from one zone to another. Hemocapillaries located along the adrenocorticocytes of the reticular zone had poorly defined lumen profiles.

The noted small thickness of the reticular zone, the small number of its cells, as well as weak blood supply to the hemocapillaries reflected a low level of steroidogenesis in the adrenal cortex. This naturally corresponded to the state of the reproductive organs hypofunction and perhaps in prospect could delay their future development. Significant expansion of venous sinus profiles was noted in the expanded medullary region of the organ $(124.6 \pm 8.5 \mu \mathrm{m})$. The collagen and reticulin bundles forming the stroma had a fibrous structure. Pycnomorphic cells prevailed among the few fibrocytes.

Small in number catecholaminosynthetic cells in the medullary region were petty, with sharply clarified cytoplasm and small rounded adrenocyte nuclei. Larger basophilically stained noradrenocytes were in significantly smaller numbers. Venous sinusoids of the cerebral substance were sharply expanded.

Consequently, the inflammatory processes increase in the gastrointestinal tract in patients with acute calves' invasion, the resorption of toxic, vasoactive metabolic products contributed to a delay in the regeneration and cellular differentiation of cortical endocrinocytes. A sharp suppression of the biosynthesis of mineral corticoids, sex steroids was noted. The weakening of glucocorticoid biosynthesis was preceded by their abundant excretion into hemocirculation in the acute period of infection. Their stimulating effect on metabolism significantly activated catabolic processes. The lethal outcome came quickly in view of the limited adaptive and plastic resources of the body of newborn sick calves in the acute course of cryptosporidiosis.

Sick calves with a chronic form of cryptosporidiosis had changes in the adrenal glands on the 15-20th day of the disease, aimed at depleting the resources of adaptive hormone production. A prolonged course of invasion contributed to a sharp expansion of the cortical substance; as a result, the thickness ratio of the cortex to the medulla was 2.5:1. The resulting structural adjustment of the adrenal cortex of sick calves was determined by the mobilization of all reserve capabilities of the body. First of all, it was manifested by prolonged hyperplasia of the cells of the fascicular zone. A significant increase in its thickness, reaching $255.7 \pm 15.9$ $\mu \mathrm{m}$, indicated this. The predominance of secretory cells over the content of spongiocytes with a pronounced foamy structure of the cytoplasm, both in the outer and inner parts of the fascicular zone, indicated a functional overstrain of adrenocorticocytes in the fascicular zone. The proportion of cells with signs of apoptosis significantly increased compared with the adrenal glands of calves with acute invasion in the chronic course of invasion in the fascicular zone. Nevertheless, manifestations of necrobiosis were noted less frequently. The majority of surviving adrenocorticocytes belonged to secret type cells due to functional overstrain, had a reduced cytoplasm volume that did not have a foamy structure. The nuclei of these cells also stood out with signs of reduced synthetic activity. The depletion of glucocorticoid biosynthesis resources was simultaneously combined with uneven blood supply in the surface layers of the cortex. Weak blood supply to the capillary net was found in deep areas especially in the medulla. Hyperemia of hemocapillaries and venous sinusoids was constantly noted.

Signs of delayed mitotic activity of cells persisted in the glomerular zone of the cortex and especially in the subcapsular region of the adrenal gland. As a result, even after 3 weeks, the glomerular zone remained poorly formed; cellular arcades did not form in it. The width of this zone was $15.3 \pm 4.2 \mu \mathrm{m}$. Cells with a foamy structure of the cytoplasm in this zone of the cortex remained single, pycnomorphic nuclei, dark oxyphilic staining of a small cytoplasm prevailed.

Consequently, the production of mineral corticoids in the adrenal cortex continued to decline in the chronic course of infection. The growing deficiency of sodium ions in the body intensified exicosis manifestations in the body of sick calves.

The reticular zone also had a significantly smaller thickness of $22.4 \pm 2.9 \mu \mathrm{m}$ and was formed by cells with basophilic staining of the cytoplasm. It was separated from the medullary substance of the organ by a more developed internal connective tissue capsule. Lumens of numerous capillaries and pericapillary spaces of the reticular zone were expanded.

A part of the adrenocorticocytes of this zone penetrated into the fascicular zone. Capillaries located along the adrenocorticocytes of the reticular zone had poorly defined lumen profiles. The small thickness of the reticular zone, the paucity of its cells, as well as the weak blood supply to the hemocapillaries reflected a low level of steroidogenesis in the adrenal cortex.

Hyperemia of venous sinuses was noted in a reduced $(96.6 \pm 12.4 \mu \mathrm{m})$ medullary substance of the organ. Small in number catecholaminosynthetic cells were represented mainly by small adrenocytes with enlightened cytoplasm and small rounded nuclei. Larger noradrenocytes were contained in much smaller quantities. The bundles proliferation of collagen and reticulin fibers in the medulla, sparseness of catecholaminosynthetic cells, and 
sharp hydroxyphilia of adrenocyte cytoplasm indicated a lower level of hormone excretion biosynthesis.

Consequently, the increase in inflammatory processes in the gastrointestinal tract of sick calves, the resorption of toxic, vasoactive metabolic products contributed to the inhibition of the biosynthesis of mineral corticoids, glucocorticoids, and sex steroids. The lethal outcome came quickly in view of the limited plastic resources of the body of newborn sick calves.

The revealed structural disorders in the adrenal glands in the form of a mitotic activity slowdown and the cells differentiation of the regenerative zone of the cortical substance reflected a low functioning level of the body adaptive mechanisms of chronically sick calves with cryptosporidiosis. The low functioning level also refers to prolonged, depleting secretion of glucocorticoids, and an increase in the apoptotic forms of adrenocorticocytes in the chronic course of infection.

The microstructure of the thyroid gland in 10-day-old calves with the acute course of cryptosporidiosis was characterized by the presence of a large number of small polymorphic follicles containing a colloidal mass of pale oxyphilic color, without the presence of resorption vacuoles near the apical region of thyrocytes being low in height. The follicular epithelium was cubic, sometimes oval, with an average height of $6.7 \pm 0.22 \mu \mathrm{m}$. The presence of only numerous clusters of secretory cells with a rounded cytoplasm and a relatively large nucleus, without signs of colloidal secretion, was noted in many sections of the organ section. Single (2-4 pcs.) parafollicular (C-cells) separated from the colloid by a significant layer of thyrocytes were found mainly in the middle part of the organ near small follicles. Signs of a biosynthesis weakening both in the cytoplasm and nucleus were also noted in relatively large parafollicular cells.

The detected presence of only single C-cells producing calcitonin indicated an increased need in calcium ions for sick calves due to insufficient intake from the inflammatory intestinal canal. Marked changes in the thyroid parenchyma corresponded to the pronounced hypofunctional state of the organ. The latter was associated with both alimentary deficiency of iodine ions in the body, and secondary causes induced by metabolic disorders as a result of the gastrointestinal tract damage of calves with acute form of cryptosporidiosis.

The thyroid gland in 15-25-day-old calves with a chronic course of cryptosporidiosis invasion was characterized by a cluster of heterogeneous in size, mainly small (65.0-60.0 \%) and a smaller number (35.0-40.0\%) of large follicles. The follicular colloid was pale oxyphilic, uniform in color, indicating a decreased level of thyroglobulin production by epithelial cells in the subcapsular region of the cortex. The follicular epithelium was significantly flattened of cubic, in places oval form, with an average height of $4.7 \pm 0.29 \mu \mathrm{m}$. Numerous clusters of rounded thyrocytes and a small rim of the cytoplasm around the hyperchromic nucleus were located in many areas (joints of the follicles). Signs of colloidal secretion were not found. The marked decreased stocks of the hormonal reserve in the follicle cavities are associated with a decrease in the needs of sick animals with a chronic course of cryptosporidiosis in thyroid hormones.

Single or 1-3 pieces of parafollicular cells (C-cells) separated from the colloid by a layer of thyrocytes were barely detected between the follicles. Signs of colloidal mass absorption in the form of resorptive vacuoles were absent in all follicles. The noted pathology is determined by a deficiency of iodine ions in the calves' body and, consequently, a low level of thyroid hormone production.

\section{Conclusion}

Significant signs of pathological metamorphosis that go beyond the adaptive reactions of the body were found in the studied peripheral endocrine organs in calves with acute and chronic cryptosporidiosis. The biosynthesis of steroid hormones by adrenocorticocytes of the adrenal cortex was impaired in the endocrine organs of sick calves. Conditions of hypofunction of thyrocytes in thyroid, and insulocytes in pancreas arose.

Identified disorders of the structure of endocrine organs were the result of primary damage to the organs of the gastrointestinal tract in the acute course of invasion. They were manifested by pathomorphological signs of a biosynthesis weakening and the removal of hormonal products into the blood circulation, accompanied by signs of endocrinocytes apoptosis. Pathological changes in the endocrine organs of sick calves acted as determining factors in the severity and outcome of the disease in a chronic infectious process.

Chronic cryptosporidiosis led to depletion of the reserves of adaptive hormone biosynthesis in endocrinocytes of the studied organs, disrupted the mitotic activity of cambial cells, and delayed cell differentiation. Moreover, autoselection of affected endocrinocytes was enhanced by the mechanism of apoptosis and necrobiosis.

Severe manifestations of the diarrhea clinic in calves during this invasion were caused both by the primary local pathogenic effect of cryptosporidium oocysts and by a disturbance of the production mechanism and adrenocorticocytes excretion of glomerular cortex into the hemocirculation of mineral corticoids. The resulting lack of sodium ions complicated the growing exicosis of the sick calf body. The established prolonged elimination of glucocorticoids into the hemocirculation from the adrenal cortex during the invasion contributed to the activation of catabolic processes in the body of sick calves, and disrupted the mechanisms of proteosynthesis. The mass death of calves with cryptosporidiosis came quickly in view of the limited energy and plastic resources of sick animals.

\section{References}

1. V.V. Zhurenko, N.M. Soroka, E.V. Zhurenko, Changes in the immunological parameters of the calves blood with cryptosporidiosis, Ecol. and Animal World, 2, 22-26 (2016) 
2. I.N. Zalialov, O.T. Mullakaev, Pathomorphology of the lymphoid organs of calves with cryptosporidiosis invasion, Sci. Notes of the Kazan State Acad. of Veter. Med. named after N.E. Bauman, 240, 93-97 (2019)

3. N.G. Iskenderova, G.D. Gaibova, Distribution of causative agents of coccidiosis of farm animals in Azerbaijan, Int. Sci. Agricult. J., 2, 54-58 (2019)

4. D.V. Kiselev, Features of complex therapy of intestinal invasions, infestations of piglets and calves, Int. Res. J., 04(58), 38-41

5. E.S Klimova, M.E Mkrtchian, T.V. Babintseva, Seasonal dynamics of calves invasion by cryptosporidiosis, Theory and Pract. of Parasitic Disease Control, 20, 273-277 (2019)

6. M.D. Novak, S.V. Engashev, R.Iu. Dzhalilov, Cryptosporidiosis - opportunistic calf invasion, Theory and Pract. of Parasitic Disease Control, 17, 296-299 (Moscow, 2016)
7. M.D. Novak, S.V. Engashev, E.Kh. Daugalieva, Theoretical and applied aspects of monitoring and preventive measures for parasitic diseases in the dairy complex, Theory and Pract. of Parasitic Disease Control, 17, 293-295 (Moscow, 2016)

8. L. Redkozubova, Cryptosporidiosis in calves, Animal Husbandry of Russ., 51-52 (February 2018)

9. A.A. Romanov, Study of parasitic diseases influence of the digestive tract on the animal organism, Territ. of Sci., 5, 7-12 (2017)

10. O. Mullakaev, I. Zalaylov, E. Kirillov, I. Konstantinova, E. Bulatova, Morphological parameters of blood of calves with cryptosporidiosis after applying different treatment regimens, BIO Web of Conferences, 17, 00031 (2020) 